\title{
Does Type of Pain Predict Pain Severity Changes in Individuals With Multiple Sclerosis? A Longitudinal Analysis Using Generalized Estimating Equations
}

\author{
Shahnaz Shahrbanian*1,2iD, Pierre Duquette ${ }^{3}$, Nancy E.Mayo ${ }^{2,4}$ \\ 1. Dept. of Sport Sciences, Faculty of Humanities, Tarbiat Modares University, Tehran, Iran \\ 2. School of Physical \& Occupational Therapy, Faculty of Medicine, McGill University, Montreal, Canada \\ 3. Notre-Dame Hospital (CHUM), University of Montreal, Montreal, Canada \\ 4. Division. of Clinical Epidemiology, McGill University Health Center, Royal Victoria Hospital, Montreal, Canada
}

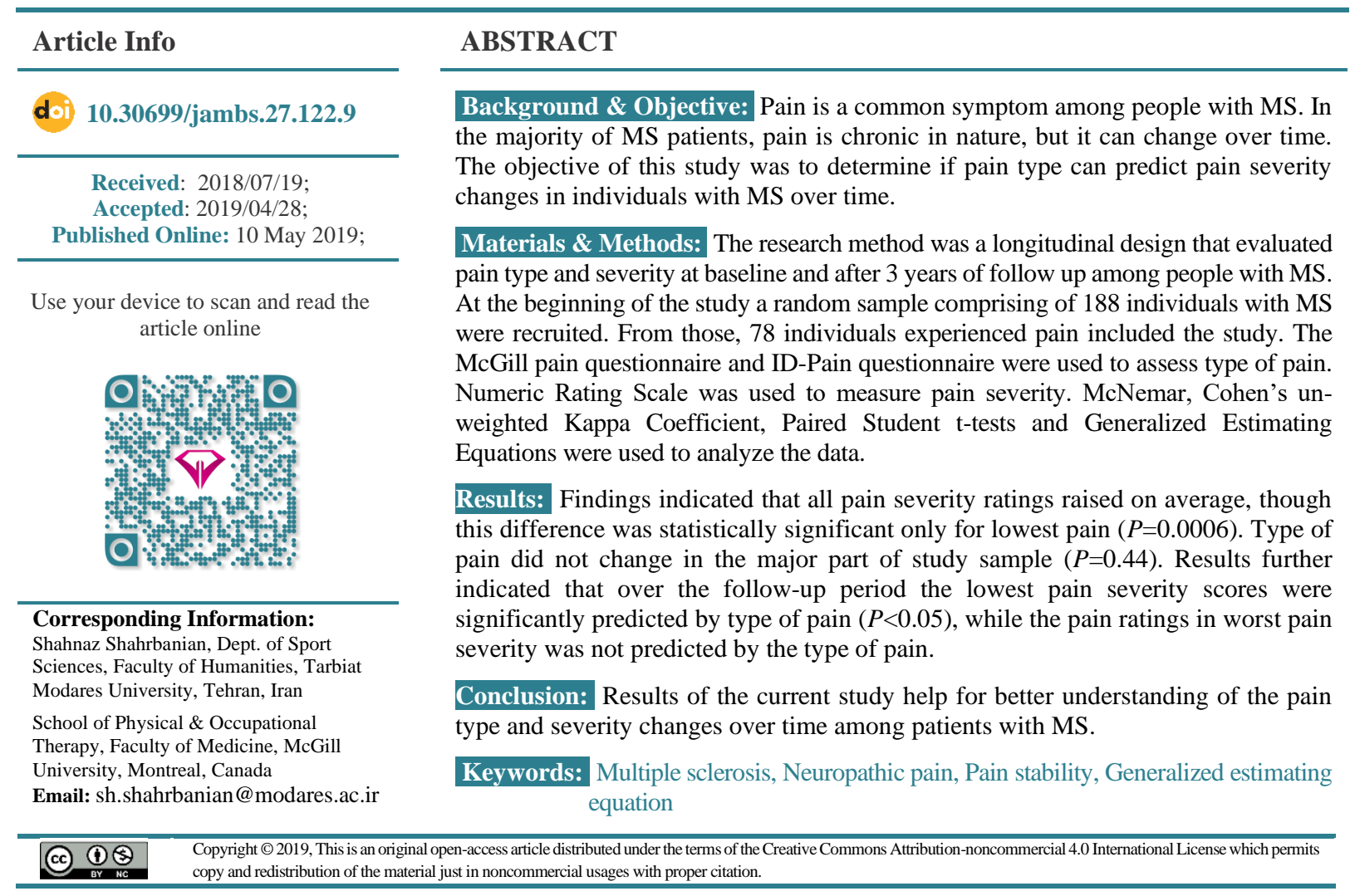

\section{Introduction}

Multiple Sclerosis (MS) is one of the most disabling chronic disease of the Central Nervous System) among young adults (1). The prevalence of MS is high in Canada (2). Pain is a common symptom in persons with MS. Pain is a complex symptom (3-6) and this complexity emphasizes the need for a multidimensional assessment. MS pain can be neuropathic pain (NP), or nonneuropathic pain $(\mathrm{N}-\mathrm{NP})(\mathbf{7 , 8})$. The damaged nerves can also evoke a range of abnormal sensations such as numbness, burning, shooting, and stabbing which are considered to be as indicators of NP (9). NP is more prevalent and disabling than N-NP in the MS population $(\mathbf{1 0 , 1 1 )}$. Also, there is evidence that the presence and severity of NP is associated with greater interference in a number of important health-related quality of life (HRQL) domains (12).
Pain impacts people both mentally and physically. A previous meta-analysis has confirmed that people with MS and pain experience a lower employment rate than people with MS who experience no pain (13). Furthermore, pain affects sleep quality and enjoyment of life in almost fifty percent of people with MS and pain (14).

The majority of MS pain is chronic in nature, but it can change over time. Given the impact of pain on the lives of people with MS, (15) the accurate assessment of changes in pain over time has become increasingly important in rehabilitation science when interpreting results of clinical studies. However, there are still questions in the literature that remained unanswered. Pain stability in MS is not a common topic of research and hence it is not adequately understood.

The main purpose of this study, was to determine the extent to which, pain type predicts pain severity changes 
over time among individuals with MS. The secondary objective was to estimate the extent to which, among individuals with MS, pain type and severity change over a follow up period of 3 years.

\section{Materials and Methods}

\section{Design}

This was a longitudinal study assessing participants' pain type and severity at baseline assessment and over a follow up period of 3 years.

\section{Participants and Procedure}

The sample population for this study consisted of 188 men and women with a diagnosis of MS since 1995 based on McDonald Criteria, registered at the three major MS clinics in the Greater Montreal, including Montreal Neurological Hospital (MNH), Centre Hospitalier de l'Universite' de Montreal (CHUM), and Clinique Neuro Rive-Sud (CNRS). The reason for including patients diagnosed since 1995, was based on MS natural history, severity of symptoms and progression of disability. Since 1995, advances in neuro-imaging techniques and disease modifying therapies (DMTs) have facilitated earlier diagnosis of disease and reduce the speed of disease progression (16). Therefore, the recognition of this era is an important methodological requirement in designing studies in this field as those people with MS diagnosed after 1995 likely not follow the same course as people diagnosed with MS prior to 1995 (16).

From those 188 individuals, 78 who reported pain were included as study participants. If participants experienced a relapse in the previous month of study and if they were younger than 18 years old, they were excluded from the study. In addition, participants with severe cognitive impairments, and those with health conditions that could impact function, such as mental illness, heart disease, rheumatoid arthritis, malignancy, renal failure, HIV/Aids, or liver failure were excluded from participating in the study as well. In order to follow-up the pain experience of this sample, after three years of first assessment, participants reporting pain at the first assessment $(n=78)$ were re-contacted to answer and complete a questionnaire on pain severity and type; 56 persons agreed to be reinterviewed.

\section{Measurement}

Socio-demographic and disease-related variables (e.g. MS type, years since MS diagnosis and symptoms onset) were collected. The severity of neurological impairment was assessed by the treating neurologists and reported according to the Expanded Disability Status Scale (EDSS) (17).

\section{Pain Type}

At baseline, participants were asked to choose as many as of the words that applied to them from a list containing the most common pain sensation descriptors taken from the McGill Pain Questionnaire (MPQ) (18). Sensations such as tingling, numbness, shooting, stabbing, electric shock-like, and burning are considered as NP descriptors, while other particular sensory adjectives such as throbbing, gnawing, or grating are significantly considered as N-NP descriptors $(19,20)$. The ID-Pain Questionnaire (ID-Pain), which is designed for accurately detecting NP as differentiated from N-NP pain, was used to assess type of pain at follow up (21). The psychometric properties of ID-Pain have been reported (21).

\section{Pain Severity}

Zero to 10 Numeric Rating Scale (NRS) was applied to assess the lowest, and worst pain severity over the last week and pain at the time of assessment (22) The reliability and validity of NRS have been reported (22-24). NRS was completed at two points in time (baseline and follow up) to estimate change in pain severity.

\section{Statistical Methods}

Descriptive statistics were used to characterize the sample. To determine the stability of the proportion of NP pain type over time, we compared the overall agreement on the classification of NP based on the results of MPQ and ID-Pain using the McNemar's test (25). To measure the agreement between within-person recorded changes in pain type over time, Cohen's un-weighted Kappa Coefficient was calculated (26). Paired Student t-tests was used to examine if serial ratings of NRS were stable or changed over time. Longitudinal analysis using generalized estimating equations (GEE) was performed to test change in lowest and worst pain severity between the different pain type groups at the first assessment and follow up (27). GEE models are extensions of generalized linear models, where outcomes for each record are not assumed to be independent (27). Participants were categorized into five groups according to the presence of NP including the reference group, the group with participants who had NP at any time point, group of participants who lost their NP at follow up, group of participants who developed NP at follow up, and group of participants who had NP at both time points. The reference group was study participants who did not have NP at either time point. Statistical analyses were performed using the Statistical Analysis Systems (SAS) Version 9.2.

\section{Results}

Of the 78 participants who reported pain in the original study, 56 persons $(72 \%)$ agreed to participate in the second phase of the study. Socio-demographic and clinical characteristics of participants and nonparticipants are presented in Table 1.

Table 2 summarizes pain characteristics of the participants. Overall, the result of McNemar's test was not significant $(P=0.44)$, indicating that there was no difference between type of pain over time (Table 2). Results of Kappa test also indicated that there was an agreement between MPQ and ID-Pain (Kappa=0.50).

The participants' descriptions of their abnormal sensations using the words from the MPQ and ID-Pain 
are also detailed in Table 2. The words most frequently used were numbness, tingling, burning and shooting, indicating that NP was the most reported type of pain. As it is presented in Table 2, there was no significant difference in the proportions of specific NP quality descriptors from MPQ and ID-Pain at first assessment and follow up.

Other data expressed in Table 2 is the mean values of participants' responses to the global pain severity scales. Results of paired t-test on serial measure of NRS showed that on average all ratings have been increased, though this difference was statistically significant only for lowest pain $(P=0.0006)$.

Table 3 presents the comparison of pain severity measured by NRS across different patterns of pain type over time. Interestingly, a descriptive analysis of the
NRS scores revealed discrepancies between change in pain type and the numerical change in pain severity scores. In comparison to reference group (participants who did not have NP at any time point), participants who had NP at any time point showed the highest increases in the current, lowest, and worst pain severity scores.

Results of a GEE analysis suggested that pain type was a significant predictor of lowest pain severity scores over the follow-up period $(P<0.05)$. Participants with NP at either time point had 1.5 points higher amount of lowest pain intensity score than those without NP (CI 95\%: 0.68-2.3). In contrast, when modeled, type of pain did not emerge as a significant predictor of worst pain rating (Table 4).

Table 1. Characteristics of the sample at the first assessment

\begin{tabular}{|c|c|c|c|c|}
\hline Variables & $\begin{array}{l}\text { Pain full sample at } \\
\text { recruitment }(\mathrm{n}=78)\end{array}$ & $\begin{array}{l}\text { Participated in the } \\
\text { follow up }(n=56)\end{array}$ & $\begin{array}{l}\text { Not participated } \\
\qquad(\mathrm{n}=22)\end{array}$ & P-value \\
\hline Age; Mean \pm SD & $44 \pm 9.9$ & $45 \pm 9.8$ & $43 \pm 8.2$ & 0.2 \\
\hline $\begin{array}{l}\text { Gender; N (\%) } \\
\text { Women }\end{array}$ & $63(81)$ & $46(82)$ & $17(77)$ & 0.2 \\
\hline Men & $15(19)$ & $10(18)$ & $5(23)$ & \\
\hline \multicolumn{5}{|l|}{ MS type; N (\%) } \\
\hline Relapsing-Remitting & $41(84)$ & $26(81)$ & $13(86)$ & 0.1 \\
\hline Secondary progressive & $4(8)$ & $3(9)$ & $1(7)$ & 0.5 \\
\hline Primary progressive & $2(4)$ & $2(6)$ & $1(7)$ & 0.7 \\
\hline Primary relapsing & $2(4)$ & $1(3)$ & 0 & 0.2 \\
\hline Years since diagnosis; Mean \pm SD & $2.9 \pm 4.9$ & $3 \pm 4.4$ & $2.4 \pm 5.9$ & 0.07 \\
\hline Years since symptom onset; Mean \pm SD & $8.8 \pm 5.3$ & $8.8 \pm 5.1$ & $9.7 \pm 5.9$ & 0.09 \\
\hline MS severity (EDSS: median, IQR*) & $3(3.5)$ & $3(3.2)$ & $2.5(2.1)$ & 0.3 \\
\hline \multicolumn{5}{|l|}{ Disease modifying therapy } \\
\hline Yes; N (\%) & $68(82.5)$ & $49(83)$ & $14(87)$ & 0.3 \\
\hline \multicolumn{5}{|l|}{ Employed } \\
\hline Yes; N (\%) & $38(48.7)$ & $26(46.4)$ & $10(45)$ & 0.5 \\
\hline
\end{tabular}

*IQR= inter quartile range 
Table 2. summarizes pain characteristics of the participants.

\begin{tabular}{|c|c|c|c|c|}
\hline Variables & $\begin{array}{l}\text { Pain full sample, at } \\
\text { recruitment }(n=78)\end{array}$ & $\begin{array}{l}\text { Pain at recruitment } \\
\text { among those with } \\
\text { follow-up }(\mathrm{n}=56)\end{array}$ & $\begin{array}{l}\text { Pain at follow-up } \\
\qquad(\mathrm{n}=56)\end{array}$ & P-value \\
\hline \multicolumn{5}{|c|}{ Pain Severity, NRS: $\bar{x} \pm S D$} \\
\hline Lowest Pain & $2.2 \pm 2$ & $2.2 \pm 1.7$ & $3.3 \pm 2$ & 0.0006 \\
\hline Worst Pain & $6.8 \pm 2$ & $7 \pm 2$ & $7.5 \pm 1.9$ & 0.1 \\
\hline Current Pain & $3.3 \pm 2.3$ & $3.3 \pm 2.2$ & $3.7 \pm 2.6$ & 0.3 \\
\hline \multicolumn{5}{|l|}{ Type of pain, $N(\%)$} \\
\hline Neuropathic & $25(37)$ & $20(36)$ & $16(30)$ & 0.44 \\
\hline Non- neuropathic & $9(13)$ & $10(18)$ & $11(20)$ & \\
\hline \multicolumn{5}{|c|}{$\begin{array}{c}\text { Pain quality descriptors, } N \\
(\%)\end{array}$} \\
\hline Pin and needles & $29(37)$ & $30(44)$ & $26(46)$ & 0.2 \\
\hline Burning & $22(29)$ & $23(34)$ & $30(56)$ & 0.06 \\
\hline Numbness & $36(46)$ & $38(56)$ & $38(68)$ & 0.37 \\
\hline Electric shock & $21(27)$ & $25(37)$ & $18(32)$ & 0.4 \\
\hline
\end{tabular}

NRS= 0-10 Numerical Rating Scale

Table 3.Comparison of pain severity measured by NRS across different groups of pain type $(\mathrm{N}=56)$

\begin{tabular}{|c|c|c|c|c|c|c|c|c|c|c|c|c|c|c|}
\hline \multicolumn{3}{|c|}{ Type of pain } & \multicolumn{3}{|c|}{ First assessment } & \multicolumn{3}{|l|}{ Follow up } & \multicolumn{3}{|c|}{ First assessment difference a } & \multicolumn{3}{|c|}{ Follow up difference ${ }^{b}$} \\
\hline 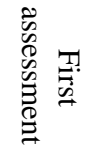 & \begin{tabular}{l}
$0^{T}$ \\
$\stackrel{\bar{O}}{0}$ \\
\multirow{\Sigma}{\Sigma}{} \\
$\tilde{\sigma}$
\end{tabular} & 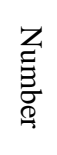 & 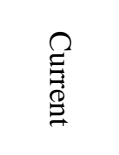 & $\begin{array}{l}5 \\
0 \\
\$ \\
8 \\
2\end{array}$ & $\begin{array}{l}\sum \\
0 \\
0 \\
0\end{array}$ & $\begin{array}{l}\hat{\Xi} \\
\Xi \\
\Xi\end{array}$ & 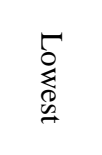 & 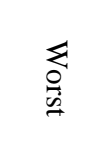 & $\begin{array}{l}\Xi \\
\Xi \\
\Xi\end{array}$ & \begin{tabular}{l}
5 \\
$\$$ \\
$\$$ \\
\hdashline
\end{tabular} & $\begin{array}{l}\sum \\
0 \\
\vdots \\
\vdots\end{array}$ & $\begin{array}{l}\hat{E} \\
\Xi \\
\Xi\end{array}$ & $\begin{array}{l}5 \\
\delta \\
\& \\
0 \\
2\end{array}$ & \begin{tabular}{l}
$\gtrless$ \\
0 \\
0 \\
\hdashline
\end{tabular} \\
\hline \multicolumn{15}{|l|}{ Stable } \\
\hline Mixed & Mixed & 14 & $2.9 \pm 2.2$ & $1.7 \pm 1.4$ & $7.9 \pm 1.8$ & $3.5 \pm 2.6$ & $2.8 \pm 1.6$ & $8 \pm 1.5$ & 0.6 & 0.9 & 1.4 & 0.7 & 0.8 & 1.9 \\
\hline NP & $\mathrm{NP}$ & 13 & $4.1 \pm 2.2$ & $2.6 \pm 1.7$ & $7.4 \pm 1.8$ & $3.7 \pm 3.4$ & $4.4 \pm 2.7$ & $7.9 \pm 1.6$ & 1.8 & 1.8 & 0.9 & 0.9 & 2.2 & 1.8 \\
\hline $\mathrm{N}-\mathrm{NP}$ & N-NP & 6 & $2.3 \pm 2.7$ & $0.8 \pm 1$ & $6.5 \pm 3$ & $2.8 \pm 2.3$ & $2 \pm 1.8$ & $6.1 \pm 2.7$ & & & & & & \\
\hline \multicolumn{15}{|l|}{ Change } \\
\hline NP & Mixed & 12 & $3.6 \pm 2.2$ & $2.7 \pm 1.5$ & $6.4 \pm 1.4$ & $4.9 \pm 1.8$ & $3.8 \pm 1.7$ & $7.2 \pm 1.9$ & 1.3 & 1.9 & -0.1 & 2.1 & 1.8 & 1.1 \\
\hline Mixed & N-NP & 5 & $3.5 \pm 1.6$ & $2.7 \pm 2.7$ & $6.5 \pm 1.9$ & $2.4 \pm 2.3$ & $2.4 \pm 1.8$ & $5.6 \pm 1.7$ & 1.2 & 1.9 & 0 & -0.4 & 0.4 & -0.5 \\
\hline $\mathrm{N}-\mathrm{NP}$ & Mixed+ Y & 4 & $3.4 \pm 0.8$ & $3 \pm 0.5$ & $7.3 \pm 1.8$ & $3.5 \pm 0.8$ & $3.3 \pm 0.5$ & $9.5 \pm 0.5$ & 1.1 & 2.2 & 0.8 & 0.7 & 1.3 & 3.4 \\
\hline Mixed & NP & 2 & $4 \pm 2.8$ & $4 \pm 1.4$ & 8 & 7 & $3 \pm 1.4$ & $8.5 \pm 0.7$ & 1.7 & 3.2 & 1.5 & 4.2 & 1 & 2.4 \\
\hline
\end{tabular}

$\mathrm{NP}=$ Participants with neuropathic pain $\quad \mathrm{N}-\mathrm{NP}=$ Participants with non-neuropathic

Mixed $=$ Participants with both neuropathic and non-neuropathic pain $\quad$ NRS=0-10 Numerical Rating Scale

a, b In comparison to reference group (participants who did not have NP at any time point) 
Table 4. Light field microscopy of the inhibitory effect of fluconazole combined with amphotericin B on fluconazole-resistant $C$. albicans (ATCC 10231) biofilm formation at different concentrations. Magnification $\times 40$, Bar=50 $\mu$ m. Flu: fluconazole, AmB: amphotericin B.

\begin{tabular}{|c|c|c|c|}
\hline Variable & Sample size & Effect & $95 \% \mathrm{CI}$ \\
\hline \multicolumn{4}{|l|}{ Lower Pain Intensity } \\
\hline \multicolumn{4}{|l|}{ All Pain Type Groups } \\
\hline No NP at any time & 6 & Referent & \\
\hline Any NP at any time & 50 & 1.5 & $0.68-2.3$ \\
\hline \multicolumn{4}{|l|}{ Post-hoc Models } \\
\hline No NP at any time & 6 & Referent & \\
\hline Lost NP at follow up & 5 & 1.13 & $-0.5-2.8$ \\
\hline Developed NP at follow up & 4 & 1.08 & $-0.43-2.6$ \\
\hline NP at both times & 41 & 1.59 & $0.74-2.4$ \\
\hline \multicolumn{4}{|l|}{ Worst pain intensity } \\
\hline \multicolumn{4}{|l|}{ All pain type groups } \\
\hline No NP at any time & 6 & Referent & \\
\hline Any NP at any time & 50 & 1.1 & $-0.78-3$ \\
\hline \multicolumn{4}{|l|}{ Post-hoc Models } \\
\hline No NP at any time & 6 & Referent & \\
\hline Lost NP at follow up & 5 & -0.3 & $-2.5-1.9$ \\
\hline Developed NP at follow up & 4 & 1.66 & $-0.8-4.2$ \\
\hline NP at both times & 41 & 1.2 & $-0.6-3$ \\
\hline
\end{tabular}

$\mathrm{NP}=$ Neuropathic pain

\section{Discussion}

This study assessed the change in pain type and severity over a follow up period of 3 years among a sample of individuals with MS. Consistent with previous studies, $(3,4,8)$ results of serial measures of NRS in this study showed that, on average, all ratings of pain severity increased, however, only lowest pain showed the significant increase. Considering the contribution of pain to problems with function in people with MS, these results emphasize on priority of early treatment and assessment of pain as it may get worse over time. The group-based analysis further showed that pain type was stable in the majority of study participants. This is in agreement with the results of study conducted by Osterberg et al. in 2005 (11) suggesting that type of pain is associated with MS duration or MS progression.

Consistent with other studies, $(\mathbf{1 0}, \mathbf{1 1})$ the results of our work confirm that NP is more common than N-NP in MS population. This emphasizes the need for adequate pain investigation, something that is still a challenging task to researchers and clinicians as there are no universally accepted diagnostic criteria for NP (23). Although, using the questionnaires have the advantage as they are fast and easy to administer, we acknowledge that a more accurate diagnosis needs to be based on patient history, and physical examination (10).

Using GEE analysis, we found that everyone who had NP at any time point had their lowest pain severity significantly higher than those who never had NP, while worst pain severity was not affected by type of pain. Having high score of lowest pain intensity is likely to be debilitating as the person is always in a state of pain.

This study had several strengths. It assessed change over time in pain type and severity which is often not assessed in MS pain literature. The present study was also the first to evaluate the predictive influence of neuropathic type of pain on pain rating over a period of three years in people with MS. Moreover, the participants in this study were randomly 
recruited from three MS clinics in different areas of the greater Montreal area, thus they were culturally diverse in MS severity, type, and gender, and so a good representative of the Canadian MS population.

As with any research, this study has limitations that should be considered when interpreting the results. First, although results of previous studies support the idea that using pain verbal descriptors can be the easiest and most reliable way to discriminate NP from N-NP, (28-30) it should be acknowledged that bias may have been introduced due to overlap in descriptors of NP and N-NP. Second, as only patients diagnosed with MS since 1995 were included in the study, this may underrepresent symptoms in study sample. Third, because of small sample size of study there would have been little power to find strong conclusion. Nevertheless, the combination of a robust statistical analysis (i.e. GEE) and the innovative approach to dealing with small sample sizes (i.e. person-specific analysis) we were able to answer the study's questions.

Findings from the current study provide useful information to help health care professionals, clinicians, and researchers to have a better understanding about NP in MS. In the clinical setting, distinguishing type of pain is essential for its treatment, since each type of pain needs specific treatment approaches according to its underlying mechanism (31). In addition, as NP is often a symptom over which clinicians cannot have significant control, maintaining an awareness of a patient's pain experience, and observing changes in serial pain measurements, increase our insight about the nature of pain type in MS and can give both patients and clinicians a greater sense of control. Considering that NP is distressing and impacts on functioning, $(\mathbf{1 0 , 1 1 )}$ this would give significant physical and psychological benefits, and so improving HRQL (12).

As found in other studies $(21,32)$, results of the present study confirm that a single measure of pain severity such as NRS alone, is an inadequate assessment tool as it may not sufficiently represent the construct of pain. Two individuals, who report same amount of pain severity on an NRS, may indicate different pain sensations. This finding has clinical implications. For treatment to be successful, measuring other aspects of pain using valid and reliable measures is necessary.

By tracking the changes in MS pain over time, the dynamic nature of pain, which is a feature that due to the unpredictability of the disease course and the interaction between pain and other symptoms has been relatively ignored in the MS pain literature, can be examined. A biopsychosocial model of pain should be considered in any assessment of pain treatment efficacy (32). This would subsequently improve the development of more effective, comprehensive treatment efforts directed toward enabling individuals with MS to maintain their active life.

\section{Conclusion}

The present study examined stability in pain type and severity among individuals with MS. While pain severity increased with time, pain type was stable. The findings of this study have practical applications for chronic pain management programs. Observing changes in serial pain measurements increases our understanding about the nature of pain in MS.

\section{Acknowledgements}

Authors acknowledge the contribution of Canadian Institutes of Health Research (CIHR) and Susan C. Scott who helped for statistical analyses and Élaine Roger, Ayse Kuspinar, and Shang Yuan Teng, who recruited patients from CHUM and $\mathrm{MNH}$, respectively.

\section{Conflict of Interest}

Authors declared no conflict of interests.

\section{References}

1. Svendsen KB, Jensen TS, Overvad K, Hansen HJ, KochHenriksen N, Bach FW. Pain in patients with multiple sclerosis. Arch Neu. 2003; 60(8): 1089-1094. [DOI:10.1001/a rchneur.60.8.1089] [PMID]

2. Archibald CJ, McGrath PJ, Ritvo PG, Fisk JD, Bahn V, Maxner CE, et al. Pain prevalence, severity, and impact in a clinical sample of multiple sclerosis patients. Pain. 1994; 58 (1): 89-93. [DOI:10.1016/0304-3959(94)90188-0]

3. Ehde DM, Gibbons LE, Chwastiak L, Bombardier CH, Sullivan MD, Kraft GH. Chronic pain in a large community sample of persons with multiple sclerosis. Mult Scler. 2003; 9(6): 605-11. [DOI:10.1191/1352458503ms939oa] [PMID]

4. Ehde DM, Osborne TL, Hanley MA, Jensen MP, Kraft GH. The scope and nature of pain in persons with multiple sclerosis. Mult Scler. 2006; 12(5): 629-38. (DOI:10.1177/1352458506071346) (PMID)

5. Piwko C, Desjardins OB, Bereza BG. Pain due to multiple sclerosis: Analysis of the prevalence and economic burden in Canada. Pain Res Manag. 2007; 12(4): 259-265. (DOI:10.1155/2007/128681) (PMID) (PMCID)

6. Von Korff M, Jensen MP, Karoly P. Assessing global pain severity by self-reports in clinical and health services research. Spine. 2000; 25(12) : 3140-3151. (DOI:10.1097/00007632200012150-00009) (PMID)

7. Beiske AG, Pedersen ED, Czujko B, Myhr KM. Pain and sensory complaints in multiple sclerosis. Eur J Neurol. 2004; 11(7): 479- 82. (DOI:10.1111/j.1468-1331.2004.00815.x) (PMID)

8. Solaro C, Brichetto G, Amato MP, Cocco E, Colombo B, D'Aleo A. The prevalence of pain in multiple sclerosis: a multicenter cross-sectional study. Neurology. 2004; 63(5): 919-21. (DOI:1 0.1212/01.WNL.0000137047.85868.D6) (PMID) 
9. Boureau F, Doubrere JF, Luu M. Study of verbal description in neuropathic pain. Pain. 1990; 42(2): 145-152. (DOI:10.1016/03 04-3959(90)91158-F)

10. Herr K. Neuropathic pain: a guide to comprehensive assessment. Pain Manag Nurs. 2004; 5 (4 Suppl 1): 9-18. (DOI:10.1016/ i.pmn.2004.10.004) (PMID)

11. Osterberg A, Boivie J, Thuomas KA. Central pain in multiple sclerosis- prevalence and clinical characteristics. Eur J Pain. 2005; 9(5): 531-42. (DOI:10.1016/j.ejpain.2004.11.005) (PMID)

12. Jensen MP, Chodroff MJ, Dworkin RH. The impact of neuropathic pain on health-related quality of life: Review and implications. Neurology. 2007; 68(15); 1178-1182. (DOI:10.12 12/01.wnl.0000259085.61898.9e) (PMID)

13. Shahrbanian Sh, Auais M, Duquette P, Andersen K, Mayo NE. Does Pain in Individuals with Multiple Sclerosis Affect Employment? A Systematic Review and Meta-analysis. Pain Res and Manag. 2013; 18 (5): e94-e100. (DOI:10.1155/ 2013/829464) (PMID) (PMCID)

14. Hadjimichael O, Kerns RD, Rizzo MA, Cutter G, Vollmer T. Persistent pain and uncomfortable sensations in persons with multiple sclerosis. Pain. 2007; 127(1-2): 35-41. (DOI:10.1016/ i.pain.2006.07.015) (PMID)

15. Lidgren L. The bone and joint decade IASP 2000-2010. Bull World Health Organ. 2003; 81(9): 629.

16. Mayo N. Setting the agenda for multiple sclerosis rehabilitation research. Mult Scler. 2008; 14(9): 1154: 6. (DOI:10.1177/13524 58508096567) (PMID)

17. Kurtzke JF. Rating neurologic impairment in multiple sclerosis: an expanded disability status scale (EDSS). Neurology. $\quad 1983 ; \quad 33(11)$ : $1444-1452$ (DOI:10.1212/WNL.33.11.1444) (PMID)

18. Melzack R, Katz J. The McGill Pain Questionnaire: appraisal and current status. In: Turk DC. New York: The Guilford Press. 1992. 152-68.

19. Victor TW, Jensen MP, Gammaitoni AR, Gould EM, White RE, Galer BS. The dimensions of pain quality: factor analysis of the Pain Quality Assessment Scale. Clin J Pain. 2008; 24(6): 550-5. (DOI:10.1097/AJP.0b013e31816b1058) (PMID)

20. Wilson M. Overcoming the challenges of neuropathic pain. Nurs Stand. 2002; 16(33): 47-53. (DOI:10.7748/ns2002. 05.16.33. 47.c3190) (PMID)

21. Portenoy R. Development and testing of a neuropathic pain screening questionnaire: ID Pain. Curr Med Res Opin. 2006;
22(8): 1555-1565. (DOI:10.1185/030079906X115702) (PMID)

22. Jensen MP, Turner JA, Romano JM, et al. Comparative reliability and validity of chronic pain intensity measures. Pain. 1999; 83(2): 157-162. (DOI:10.1016/S0304-3959(99)001013)

23. Jensen MP. Review of Measures of Neuropathic Pain. Curr Pain Headache Rep. 2006; 10(3): 159-166. (DOI:10.1007/s11916-006-0041-z) (MID)

24. Sharrack B, Hughes RA, Soudain S, Dunn G. The psychometric properties of clinical rating scales used in multiple sclerosis. Brain.1999; 122 (1): 141-59. (DOI:10.1093/brain/122.1.141) (PMID)

25. McNemar Quinn. Note on the sampling error of the difference between correlated proportions or percentages. Psychometrika. 1947; 12(2): 153-157. (DOI:10.1007/BF02295996) (PMID)

26. Carletta Jean. Assessing agreement on classification tasks: The kappa statistic. Computational Linguistics. 1996; 22(2): 249254.

27. Hanley JA, Negassa A, Edwardes MD, Forrester JE. Statistical Analysis of Correlated Data Using Generalized Estimating Equations: An Orientation. Am J Epidemiol. 2003; 157(4): 364-375. (DOI:10.1093/aje/kwf215) (PMID)

28. Dubuisson D, Melzack R. Classification of clinical pain descriptions by multiple group discriminant analysis. Exp Neurol. 1976; 51(2): 480-7. (DOI:10.1016/0014$\underline{4886(76) 90271-5)}$

29. Hallström H, Norrbrink C. Screening tools for neuropathic pain: can they be of use in individuals with spinal cordinjury? Pain. 2011; 152 (4): 772-9. (DOI:10.1016/j.pain.2010.11.019) (PMID)

30. Padua L, Briani C, Truini A, Aprile I, Bouhassirà D, Cruccu $\mathrm{G}$, Consistence and discrepancy of neuropathic pain screening tools DN4 and ID-Pain. Neurol Sci. 2013; 34(3): 373-7. (DOI:10.10 07/s10072-012-1011-3) (PMID)

31. Moulin DE, Foley KM, Ebers GC. Pain syndromes in multiple sclerosis. Neurology. 1998; 38(12): 1830-34. (DOI:10.1212 /WNL.38.12.1830) (PMID)

32. Kerns R. Psychosocial aspects of pain. Int J MS Care. 2000; 2(4): 35-38. (DOI:10.7224/1537-2073-2.4.37)

\section{How to Cite This Article:}

Shahrbanian S, Duquete P, Mayo N. Does Type of Pain Predict Pain Severity Changes in Individuals With Multiple Sclerosis? a Longitudinal Analysis Using Generalized Estimating Equations. J Adv Med Biomed Res. 2019; $27(122): 9-15$

\section{Download citation:}

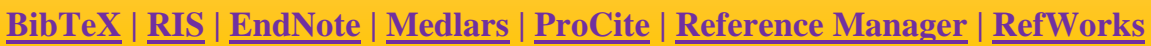

\section{Send citation to:}

(38) Mendelev 2 Zotero (i) RefWorks $\underline{\text { RefWorks }}$ 